\title{
Lithographed spectrometers for tomographic line mapping of the Epoch of Reionization
}

\author{
R. O’Brient,,${ }^{a, b}$ J.J. Bock,${ }^{a, b}$ C. M. Bradford ${ }^{b}$ A. Crites,${ }^{a, b}$ R. Duan, ${ }^{a}$ S. Hailey-Dunsheath,${ }^{a}$ J. \\ Hunasek, ${ }^{a}$ R. LeDuc, ${ }^{b}$ E. Shirokoff, ${ }^{c}$ Z. Staniszewski, ${ }^{a, b}$ A. Turner, ${ }^{b}$ M. Zemcov ${ }^{a, b}$ \\ ${ }^{a}$ California Institute of Technology, 1200 E. California Blvd., Pasadena, CA 91125 USA; \\ ${ }^{b}$ Jet Propulsion Laboratory, 4800 Oak Grove Dr., Pasadena, CA 91109, USA; \\ ${ }^{c}$ Department of Astronomy, University of Chicago, Chicago, Ill USA;
}

\begin{abstract}
The Tomographic Ionized carbon Mapping Experiment (TIME) is a multi-phased experiment that will topographically map [CII] emission from the Epoch of Reionization. We are developing lithographed spectrometers that couple to TES bolometers in anticipation of the second generation instrument. Our design intentionally mirrors many features of the parallel SuperSpec project, inductively coupling power from a trunk-line microstrip onto half-wave resonators. The resonators couple to a rat-race hybrids that feeds TES bolometers. Our 25 channel prototype shows spectrally positioned lines roughly matching design with a receiver optical efficiency of $15-20 \%$, a level that is dominated by loss in components outside the spectrometer.
\end{abstract}

Keywords: Epoch of Reionization, spectrometer

\section{ASTROPHYSICAL MOTIVATION}

The Epoch of Reionization (EoR) is an era during which ultraviolet emission from the first generation of stars in the Universe ionized the intergalactic medium in a process which was completed by $z \sim 6^{1}{ }^{2}$ Because of the distance to and intrinsic low luminosity of EoR sources, this era remains poorly understood. The Lyman- $\alpha$ break renders measurements at $\lambda \lesssim 1 \mu \mathrm{m}$ sensitive only to the end of reionization, making infrared and radio measurements of EoR emission attractive. ${ }^{3}$

Several teams, such as PAPER, LOFAR, and MWA are attempting to use the hyperfine transition in neutral hydrogen at $\lambda=21 \mathrm{~cm}$ line as a tracer of structure during and prior to the EoR. In contrast to the Lyman- $\alpha$ line, the $21 \mathrm{~cm}$ transition has an extremely low scattering cross-section so avoids the short mean free path that renders the EoR opaque to optical and near-infrared photons. By generating maps at different redshifts, these experiments can trace neutral hydrogen's density and structure over time, producing a history of reionization. ${ }^{4}$ In practical terms, these experiments engage in intensity mapping, a powerful technique in which the average emission over large regions is measured, and statistical techniques are used to infer bulk properties of the emission. ${ }^{5}$ However, challenges abound: the signal is faint, and redshifts into the range $v=150-250 \mathrm{MHz}$ where interference from galactic and commercial radio sources are a significant foreground to the measurement. Cross-correlation of $21 \mathrm{~cm}$ maps with those of other atomic lines that originate during EoR effectively removes the foregrounds from either measurement, thereby improving the accuracy and power of both. ${ }^{6}$

TIME will map ionized carbon [CII] as a complimentary means of tracing reionization history. The $158 \mu \mathrm{m}$ [CII] line has significantly longer wavelength than can interact with neutral hydrogen so should be visible to large redshifts. Additionally, because it is a significant cooling mechanism in star formation and accounts for up to $\sim 1 \%$ of the bolometric power from star-forming galaxies, the $158 \mu \mathrm{m}$ line should be bright in systems in which carbon is present. ${ }^{7}$ Furthermore, $158 \mu \mathrm{m}$ emission between $z=9$ and $z=5$ redshifts into the $200-300 \mathrm{GHz}$ atmospheric window, permitting observations from ground-based instruments that can provide the necessary arcmin resolution needed to resolve the clustered component of the spatial power spectrum. A power spectrum computed from simulation of [CII] during EoR and TIME observation of it is shown in Figure 1(a). ${ }^{8}$

We expect that $\mathrm{CO}$ lines from low-redshift galaxies will contaminate our maps, although this may be mitigated by masking maps using galaxy catalogs (see 1(a)). Ultimately, TIME and the 21-cm community can mitigate their respective foregrounds through cross-correlation, ${ }^{6}$ in which we expect spatial anti-correlation on scales larger than the typical percolation size at a given redshift (Figure 1(b)). This anti-correlation should relax to zero and eventually positively correlate on scales which are both ionized and carbon-rich, thus tracing the history of carbon emplacement in the Cosmos. ${ }^{8}$

Further author information: (Send correspondence to Roger O’Brient). E-mail: rogero@caltech.edu

Millimeter, Submillimeter, and Far-Infrared Detectors and Instrumentation for Astronomy VII, edited by Wayne S. Holland, Jonas Zmuidzinas, Proc. of SPIE Vol. 9153, 91530F · () 2014 SPIE · CCC code: 0277-786X/14/\$18 - doi: 10.1117/12.2057319 


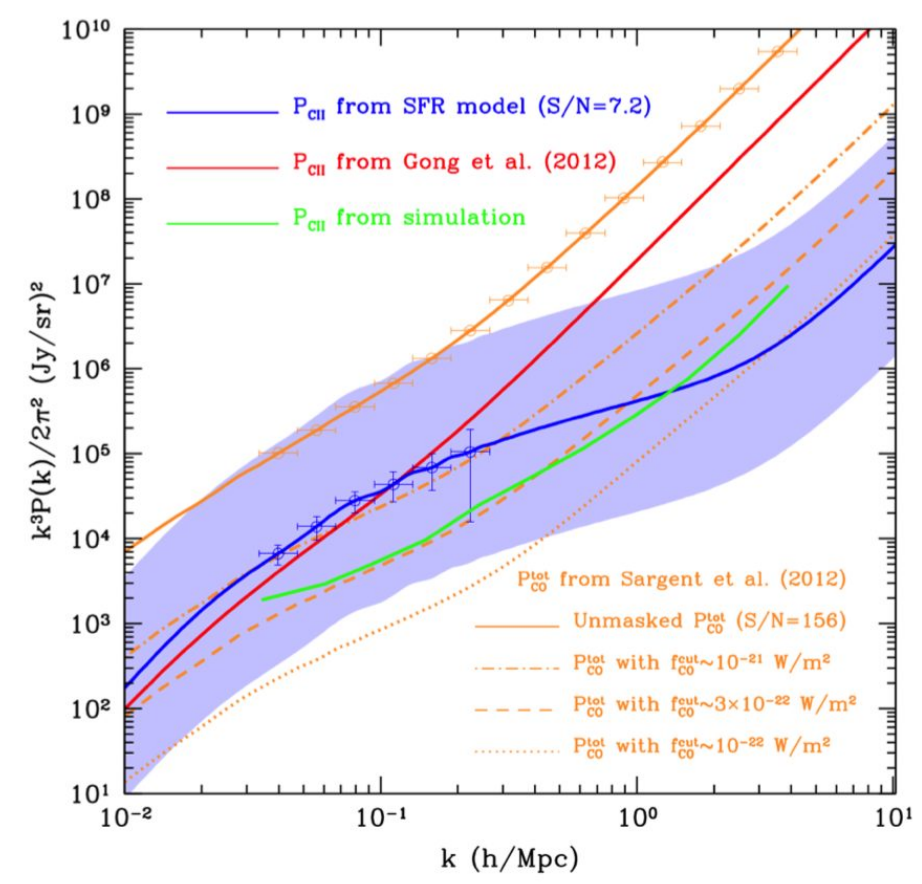

(a) Simulations of [CII] power spectrum

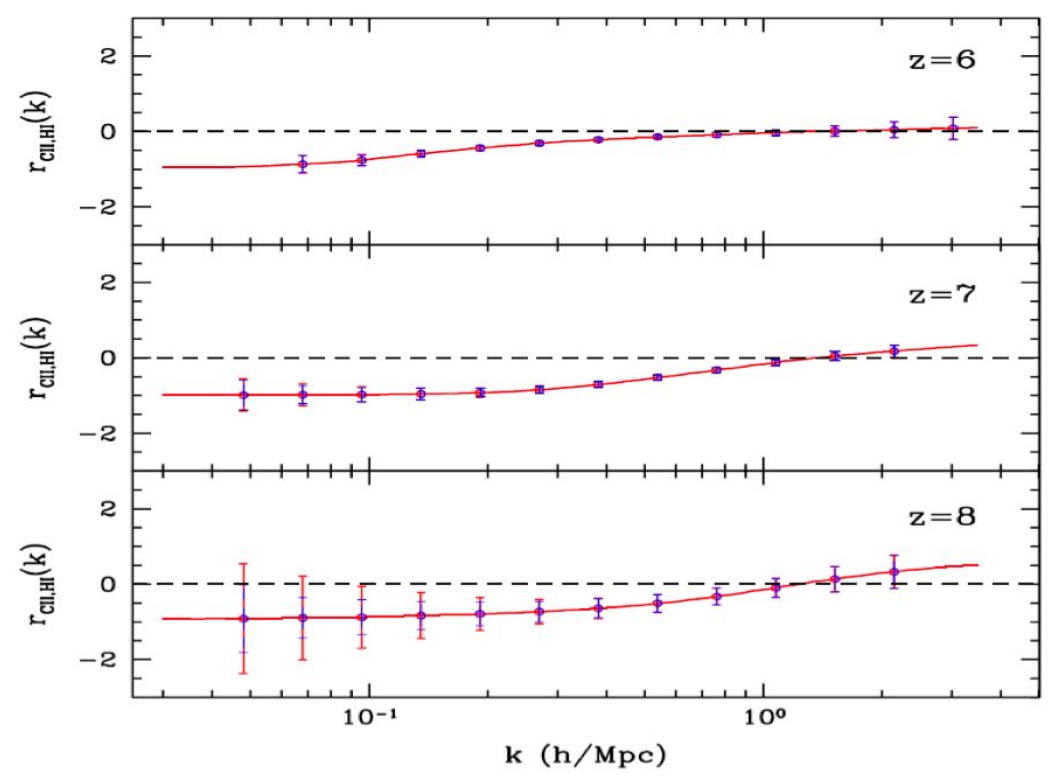

(b) Spatial cross correlation between TIME and SKA

Figure 1. Simulations of [CII] spectra using a variety of models (including projected TIME-pilot band powers and CO contamination at different filtering levels) and a simulation of spatial cross-correlation between TIME and SKA. See CITE GONG for the details of these calculations.

\section{THE TIME EXPERIMENT}

We are currently exploring a number of telescope options, including Caltech Submillimeter Observatory (CSO), Greenland Telescope (GLT), and James Clerk Maxwell Telescope (JCMT), all of which provide ample resolution $\left(\sim 0.5^{\prime}\right)$ to detect the amplitude of the [CII] power spectrum from EoR. Early attempts will scan a linear field $\theta \sim 1^{o}$ across and sensitive to spectral channels between $z=5$ to $z=9$. This strategy will ignore the second spatial direction $\phi$ so as to maximize map 
depth while still retaining the ability to differentiate 3-dimensional spatial modes that does not just rely on the spectral channel.

This first version of the TIME experiment, TIME-Pilot,${ }^{9}$ will use grating spectrometers similar to the Z-Spec instrument. ${ }^{10}$ The instrument will contain 32 spectrometers, each containing $\sim 60$ TES bolometers that span a bandwidth of 185-325GHz. For a star-formation rate sufficient to sustain reionization and [CII] brightness and metallicities matching lower redshift observations, TIME-Pilot should detect the clustering term of the [CII] spectrum with $S / N \sim 10$ after approximately a month of observing time at Mauna Kea. ${ }^{11}$ Detailed studies of the redshift history of the signal will require more sensitivity, and finer spatial and spectral resolution may be required to successfully mask out $\mathrm{CO}$ foregrounds.

TIME-Pilot uses established technology to rapidly begin observations and to begin the search for a signal. However, it is difficult to extend grating spectrometers to focal planes with both more pixels (and more per channel sensitivity) and higher resolution. We are exploring fully lithographed microstrip spectrometers in anticipation of these more sensitive cameras. Our team is investigating TES-bolometer and KID versions in parallel ${ }^{12} .{ }^{13}$ While KIDs naturally multiplex and can scale to very large arrays, their sensitivities are not yet demonstrated for this application. A TES version is a powerful hedge, where we anticipate using high MUX-factor resonator-based readout systems such as NIST's microwave MUX. ${ }^{14}$ These parallel detector lines are intentionally kept as similar as possible to allow for effective troubleshooting of designs and fabrication recipes. This paper focus on the TES-bolometer version.

\section{PIXEL PROTOTYPE}

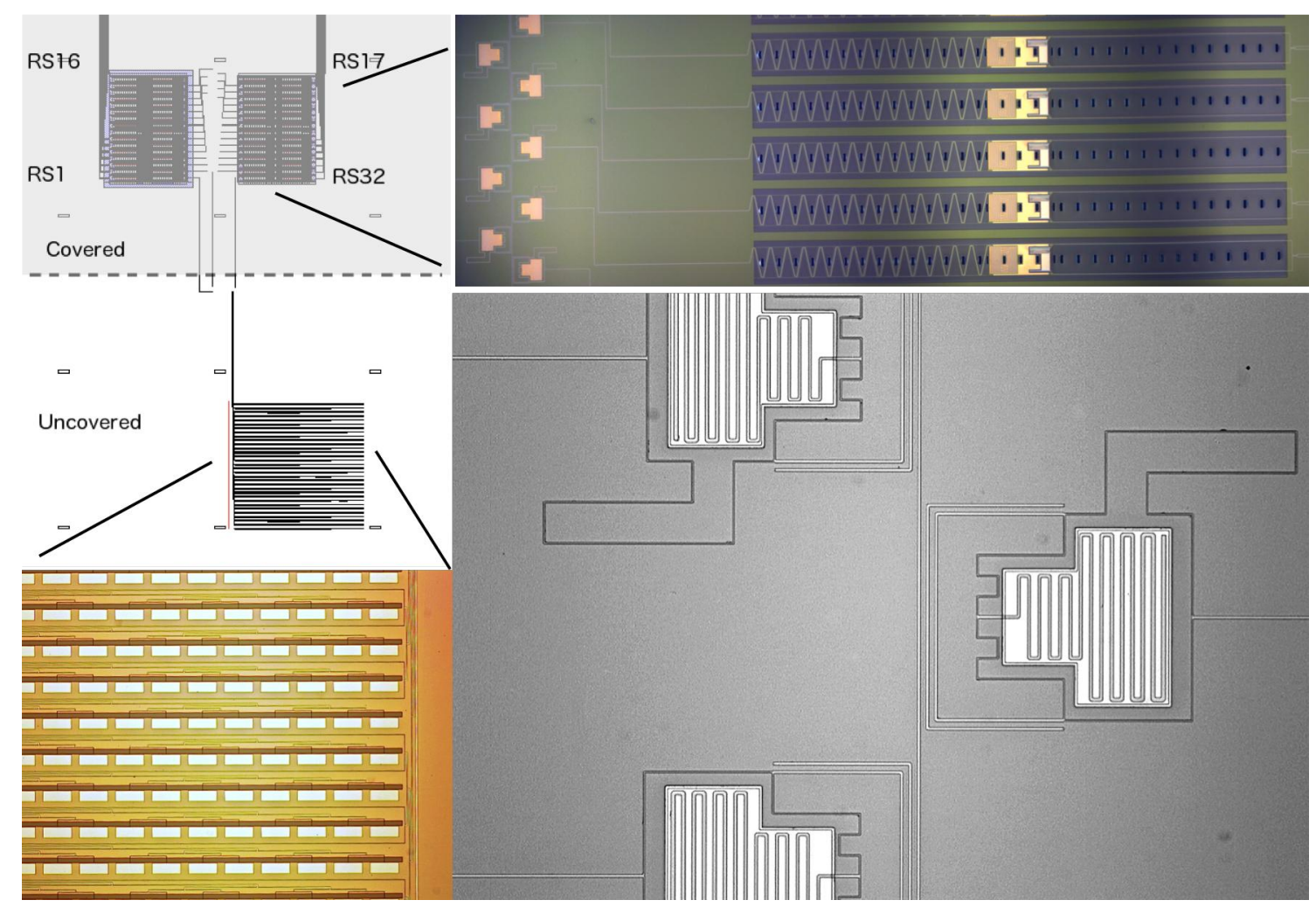

Figure 2. From top-to-bottom, left-to-right: layout of the prototype, showing covered and optically open regions; photograph of the planar phased array antenna; photograph of part of spectrometer; zoom of a few "staple" resonators, including hybrods

Our prototype uses techniques honed in the successful CMB-polarimeter detectors ${ }^{15}$ used in the BICEP2 ${ }^{16}$ and Keck ${ }^{17}$ experiments and to be used in BICEP $3{ }^{18}$ and the balloon borne SPIDER.${ }^{19}$ For these early TIME prototypes, we couple power to lithographed microstrip circuits through a singly polarized broad-band $5 \mathrm{~cm} \times 5 \mathrm{~cm}$ planar phased array antenna, similar to what has been used in the MUSIC experiment. ${ }^{20}$ Each of the $325 \mathrm{~mm}$ long slots has 32 taps fed by a corporate 
summing tree that guarantees the electric field amplitude and phase are uniform over an octave bandwidth spanning 200$300 \mathrm{GHz}$ (See Figure 2, lower left). We optimized the design in HFSS, tuning the microstrip's drive-point impedance and coupling shunt capacitor to provide less than $-10 \mathrm{~dB}$ return loss over the operating band (See Figure 3). The antennafeed performs beam synthesis and collects the octave bandwidth onto a common microstrip line that feeds power into a lithographed spectrometer.

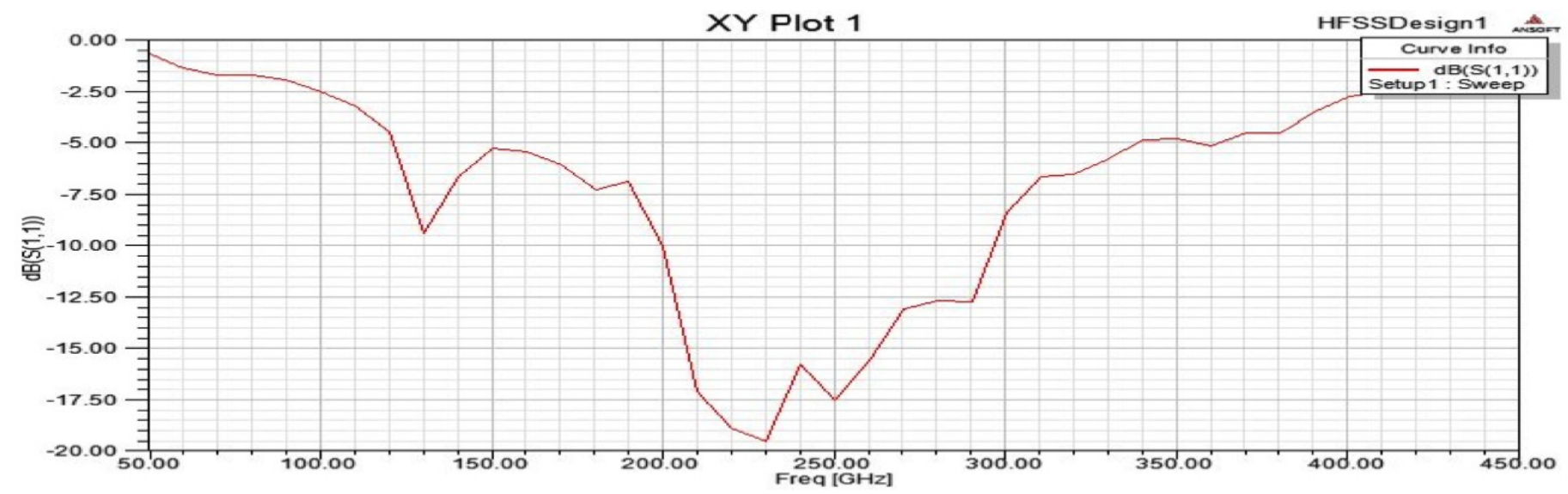

Figure 3. HFSS simulation of return-loss of antenna-feed with slots, showing acceptable performance in the 200-300GHz band. We are currently constructing devices that directly test the antenna's efficiency.

Within the spectrometer, the microstrip trunk-line inductively couples power to half-wave "staple" resonators. These resonators are arranged in order of decreasing resonant frequency and are separated from neighbors by $\lambda / 4$, ensuring that those neighbors' virtual shorts on resonance are transformed into virtual opens (See Figure 2, upper right). In contrast to SuperSpec, ${ }^{12}$ which couples power from resonators into TiN inductors, this design couples to an adjacent microstrip line, matching design $Q=300$ for coupling on each side of the resonator. Currents at the ends of the resonator are 180 degrees out of phase, so we use a hybrid to combine those waves before terminating in a lossy gold transmission on a TES bolometer island (See Figure 2, lower right).

Historically, BICEP2 and Keck bolometers received $0.5 \%$ of their optical loading through direct stimulation that bypassed the microwave circuitry. ${ }^{21}$ The design bandwidth of our spectrometer channels is roughly $0.6 \mathrm{GHz}, 2 \%$ of the $150 \mathrm{GHz}$ TES polarimeters' bandwidth. With this greatly reduced loading through the microstrip circuits, direct stimulation is proportionately larger; in an optical design similar to BICEP2, the spectrometer channels would experience $30 \%$ direct stimulation. In an effort to reduce this, we separated the antennas and spectrometers by $25 \mathrm{~mm}$ of microstrip line so that the spectrometer and bolometers are far enough from the antenna that we can shield them from direct stimulation. We do this by depositing gold on the backs of the silicon substrate and mounting a plate over the sky-side of the tile, in both cases solely over the bolometers and not obstructing the antennas. Additionally, we mounted HR10 material between the shield and AR tile to damp substrate modes. Figure 2, upper left indicates which parts of the device are optically exposed or open.

Our TES design is very similar to the SPIDER/BICEP design, with a series dual-TES of Aluminum for high-loading measurements and Titanium for lower loading. The bolometers' thin nitride legs are tuned to provide a bolometer $G \sim$ $10 \mathrm{pW} / \mathrm{K}$ and we add a thick $2 \mu \mathrm{m}$ layer of gold on the bolometer island to limit the detectors' thermal bandwidth.

\section{SPECTROMETER DESIGN}

The coupling to spectrometer resonators occurs through microstrip edge-side coupling. A similar mechanism afflicted our CMB polarimeters, inducing phase errors in our phase antennas and differentially steering polarization pairs' beams off boresight. While this effect generated systematic errors that had to be dealt with in the BICEP-2 analysis pipeline, it did provide our team a chance to measure and characterize this coupling with a range of line separations. This measurement effectively transduces the ratio of leaked power to intended power into an angular difference that could be measured precisely on a sample of several hundred devices. Reconciling measurements of a variety of line-spacings against HFSS simulations and custom quasi-static code revealed that the thickness of the upper-conducting films is an important parameter in determining coupling levels Cite ${ }^{17} .{ }^{15}$ This impacts the spectrometer Qs and must be accounted for if we are to achieve our design filter-width requirements. 
Commercially available software does not gracefully let us simulate large spectrometers, and is even slow for a single resonator. Instead, we construct scattering matrices from simple circuit models of coupled pairs of lines. This approach lets us capture the thickness of the conductors that is poorly accounted for in Method-of-Moments simulations, but also allows for simple corrections for super-conducting effects by including series kinetic inductance. We can model triples of lines by cascading the scattering matrice ${ }^{22}$ of three pairs of double lines that reflect the the three possible pairings:

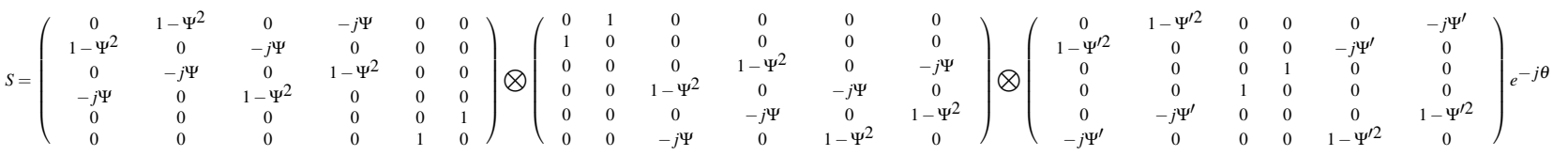

The three matrices in Equation 1 correspond to the three sections in the expanded section of Figure $4(\mathrm{a}) . \Psi=\left(k_{o}-\right.$ $\left.k_{e}\right) \ell / 2$ and reflects the difference in wavespeed between even and odd modes between adjacent modes over a length $\ell$ while $\Psi^{\prime}$ reflects this for the non-adjacent lines. The terms of these equations are taylor expansions of sin and cosine functions, so presume that $\ell \ll \lambda$. We compute the even and odd mode wavespeeds in a quasi-static finite-element algorithm implemented in Matlab that solves for the electric potential in a 2-dimensional cross-sectional plane; the model includes the upper conductors' finite thickness. The block diagonal nature of each matrix reflects how one of the three lines coupling is ignored in each matrix. By modeling the tripple-lines as a cascade of short sections 4(a), we account for power that travels between the outer lines through the middle. However, this formalism over-counts the line length by $50 \%$, so we correct this by a phase shift $\theta$. When modeling a pair of lines, as in the prongs of the staple resonators, we can simply use the upper left $4 \times 4$ sub matrix of the left most matrix in Equation 1.

The symbol $\otimes$ denotes cascading of scattering matrices. ${ }^{22}$ We model a complete resonator by cascading the linepairs for each prong onto the outer-most lines from the trunkline in the triple lines. We cascade the inner-most lines in each resonator with the hybrid who's even port is resistively terminated and who's odd is the port delivering power to a bolometer. These resonators cascade to form the entire spectrometer model.

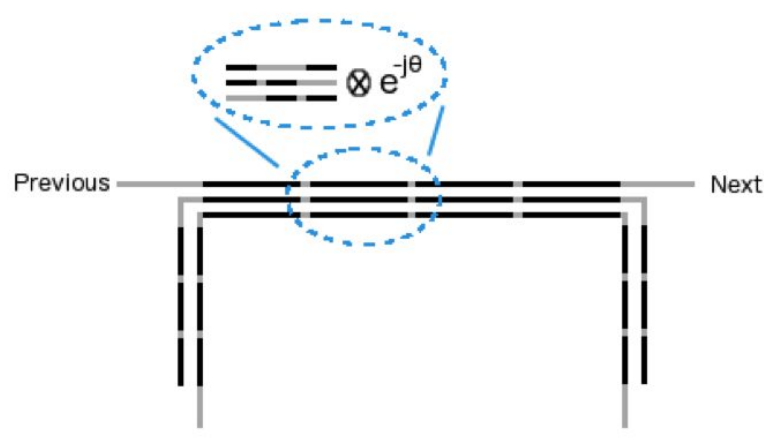

To TES Bolometer

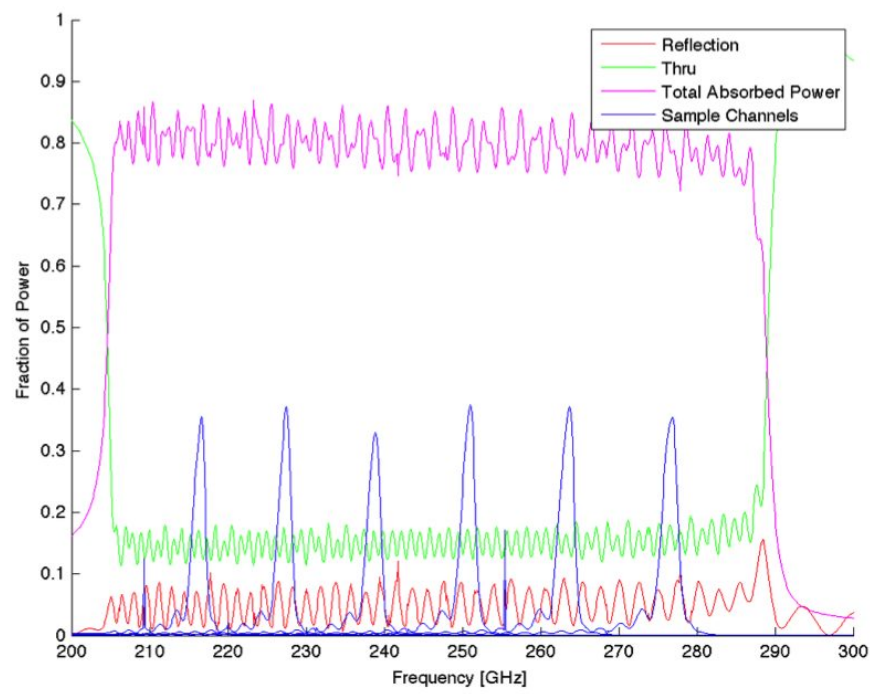

(b) Computed Scattering Parameters

(a) Circuit Model for a resonator and its decomposition into line pairs

Figure 4. Simulations of spectrometer design.

Figure 4(b) shows the results of such a computation for an 80 channel spectrometer covering the $200-300 \mathrm{GHz}$ window of interest. We only show the power transmitted to a sample of channels to avoid confusion. This early design may have a mismatch in coupling Q, which decreases the power coupling below an ideal of 50\% and widens the response to $Q \sim 200$ from a design of $Q \sim 300$. There is heavy power sharing between adjacent channels, such that the total power absorbed in each channel is $80 \%$. Around $10 \%$ of incident power is reflected and another $10 \%$ transmitted through the entire spectrometer at a given frequency. 


\section{OPTICAL CHARACTERIZATION}

Our optical test-bed was originally designed to characterize detector beams for our CMB polarimeters. The cryostat has no re-imaging lenses and locates the detectors as close to the zotefoam window as possible. It otherwise resembles the BICEP-style cameras, outfitted with a series of cryogenically cooled teflon and nylon filters as well as a metal-mesh edge filter with a $30 \mathrm{icm}(360 \mathrm{GHz})$ cutoff just above the detectors. Using an internal Helium-10 fridge, supported by a pulse-tube cooler, we cool the detectors into either $270 \mathrm{mK}$ Titanium or $1100 \mathrm{mK}$ Aluminum TES series transitions. We read our TESs through NIST's time-domain-multiplexing (TDM) system. Each prototype spectrometer fills the rows of one MUX chip (column) so the TESs share a common flux to current calibration.

We characterize the spectral response with a polarizing Martin-Puplett Fourier Transform Spectrometer (FTS), designed with an asymmetric throw length of $80 \mathrm{~cm}$, to provide $80 \mathrm{MHz}$ resolution. Misalignment of the optics can degrade interference at high displacements and compromise the fidelity of the spectra at the fine resolution scale. We employ translation and rotation stages on the beam-splitter and test lasers located at source and output points to ensure tight and repeatable alignment. It reproduces identical spectra as a lower resolution FTS and comparison to measurements with a high-Q swept local oscillator suggests that our FTS is reliable to its design resolution.

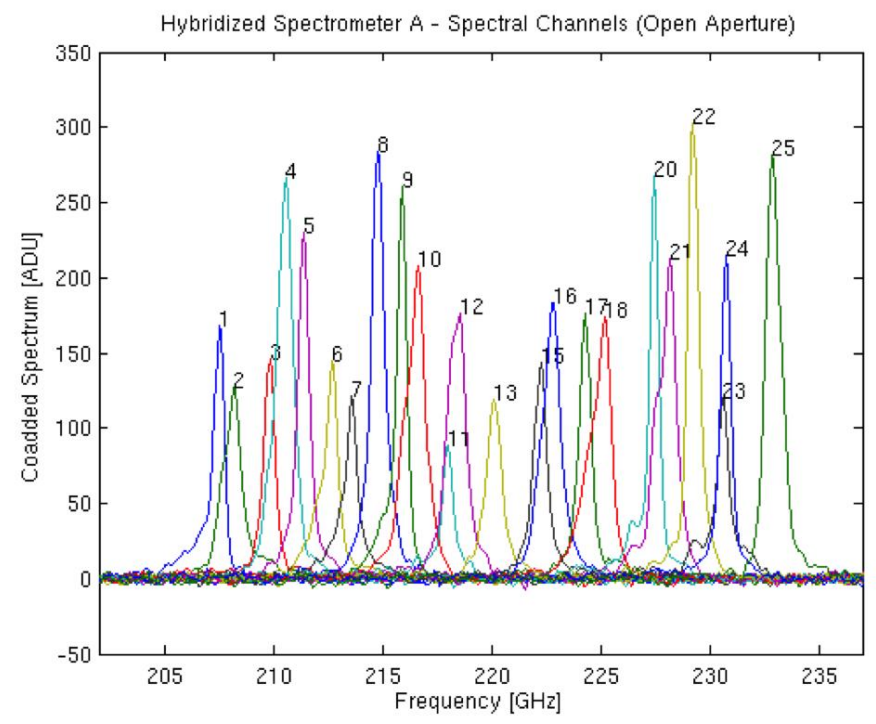

Figure 5. Prototype measurements taken with a $100 \mathrm{MHz}$ resolution FTS.

Figure 8 shows the results of FTS measurements where the vertical axis is in arbitrary units that are proportional to current through the TESes. All TESes in a spectrometer were biased into the lower noise Titanium transition and read through a common MUX chip (MUX column) with a common calibration of flux to current. The variations in height are thus physical and suggest standing wave issues, perhaps on the trunkline. These standing waves have also been seen in the KID version of the spectrometer and removing them remains an active area of research. ${ }^{23}$

Figure 6(a) shows the locations of the spectral lines, with vertical bars indicating the FWHM of the lines. This plot reveals that the line locations are monotonic with channel identity (e.g. they are in the intended order). Figure 6(b) shows a histogram of the band-widths, near the design of $0.7 \mathrm{GHz}$, corresponding to $Q \sim 300$. Note that our model slightly under-predicted the $\mathrm{Q}$ and we are refining the model to try to match the data so it can be a predictive tool for future designs.

We measure the receiver (end to end) optical efficiency of each TES bolometer with IV curves taken under loading from beam-filling black-body loads (metal backed eccosorb HR material) at $300 \mathrm{~K}$ and $77 \mathrm{~K}$. We compare the measured difference of saturation power from these two loadings against the total power difference one might expect within the measured $0.7 \mathrm{GHz}$ bandwidth, which is $2.1 \mathrm{pW}$. Figure 7 (a) shows this for three different devices. The blue curve is the power received by bolometers in a spectrometer directly fed by an antenna while the red is the power received where half of the incident power is extracted in a 5-pole quadrature hybrid circuit and delivered to a reference bolometer.

The spectrometers appear to receive more power than the $2.1 \mathrm{pW}$ per channel incident on the cryostat. The black dashed line shows the response of "dark" bolometers not fed by an antenna and spectrometer and it receives nearly as much power as the optically connected bolometers. This optical response suggests that dark stimulation contributes the dominant 


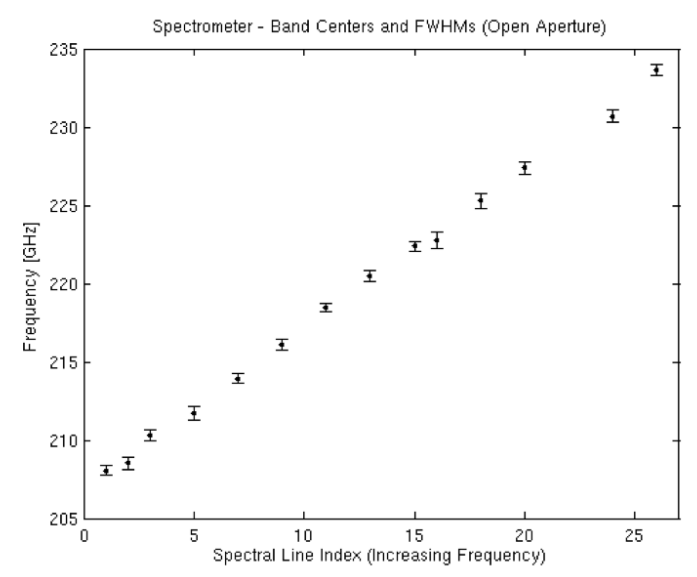

(a) Spectral locations- error bars indicate bandwidth

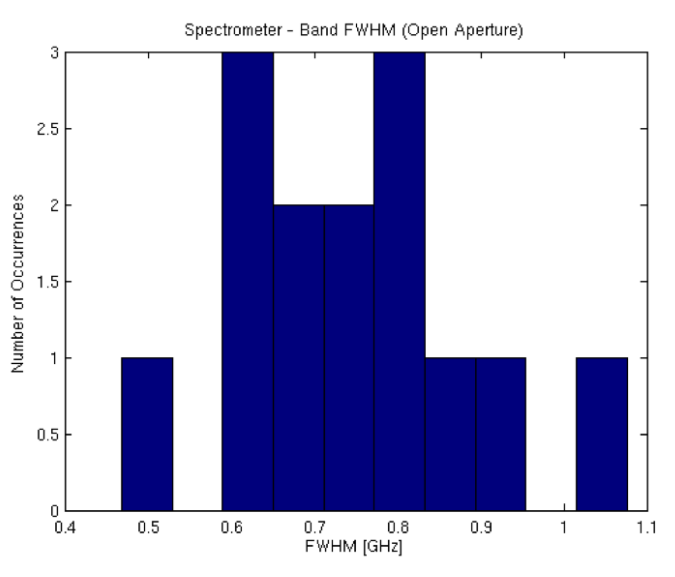

(b) Spectral Bandwidths

Figure 6. Fits parameters to lorenzian profiles of the spectrometer channels.

response to the detectors in the spectrometers. We measure a small change in the temperature of our cold stage with loading, which suggests some of this response may be due to the heating of detector tiles. However, measurements with a chopped load indicate that the dominant out-of-band response mechanism has a time constant of $\sim 10 \mathrm{~ms}$, significantly faster than has been previously measured for the tile heating in similar instruments. ${ }^{21}$ Additionally, Figure 7(a) has labels indicating the approximate distance from the edge of the cover. All three of the tested devices show a response that decreases with distance from the edge, a phenomenon poorly explained by tile heating where we expect the entire silicon substrate to be isothermal but well explained by an optical signal that experiences partial attenuation as it propagates in the lossy materials packed in front of the tile over the bolometer banks.

Dark stimulation is exacerbated in our test-bed because the focal-plane is exposed to a solid angle a factor of 4 larger than when located behind refracting optics, and our experience with BICEP2 and Keck detectors leads us to expect that these levels can be significantly reduced in a more realistic camera with reimaging optics. We are also exploring re-designs that would make the detectors intrinsically more robust against direct stimulation. These redesigns include alternate optical coupling schemes and more aggressive shielding of the bolometer banks in the detector packaging.

Expecting that this can be reduced, Figure 7(b) uses an exponential fit to the dark response from Figure 7(a) and subtracts it from the response of the directly illuminated spectrometer. We compute the ratio of this power to that of the $2.1 \mathrm{pW}$ case to form a receiver optical efficiency and find 15-20\%. The horizontal red line shows power expected in each resonator in a perfectly functioning spectrometer, where the other $50 \%$ returns to the trunk-line. The green line shows how that level is further reduced by our filter-stack and loss in our microstrip lines leading up to the spectrometer. We find that the measured efficiencies are only slightly lower than this baseline of $24 \%$. One of our goals is to directly compare against the KID devices and the details of our antenna-feed and filter stack complicate this, so comparison to the green line is in fact justified. We further note that the bolometers at row-selects 15 and 16 are dark, so low efficiency in those detectors is expected. We also plan to perform dark tests with internal thermal loads to both check that the excess power is direct stimulation and also measure efficiency without the filter stack.

Finally, we can use the spectral response of the reference bolometer in the hybridized device to normalize the spectral channels. This ratio removes all losses that are on the "sky"-side of the spectrometer (except $4 \mathrm{~mm}$ of microstrip between the hybrid and spectrometer), providing an efficiency that is more representative of the intrinsic properties of the device. In this case, we find an efficiency between 30-60\%, similar to what we might expect for resonators that absorb half of the incident power. Because we biased onto the higher saturation-power but lower sensitivity Aluminum transition to avoid saturating the heavily loaded reference bolometer, this measurement has a low signal to noise ratio.

\section{CONCLUSIONS AND FUTURE WORK}

The data from TIME will allow a reconstruction of the EoR's detailed history, but our long-term scientific goals will require higher resolution and pixel-count than can be achieved with grating spectrometers. We have demonstrated a working lithographed spectrometer that couples a $200-230 \mathrm{GHz}$ band to 30 TES bolometers with operating $Q \sim 300$ and optical efficiencies between $15 \%-20 \%$ after subtraction of direct stimulation. We note that this performance is relatively high once 


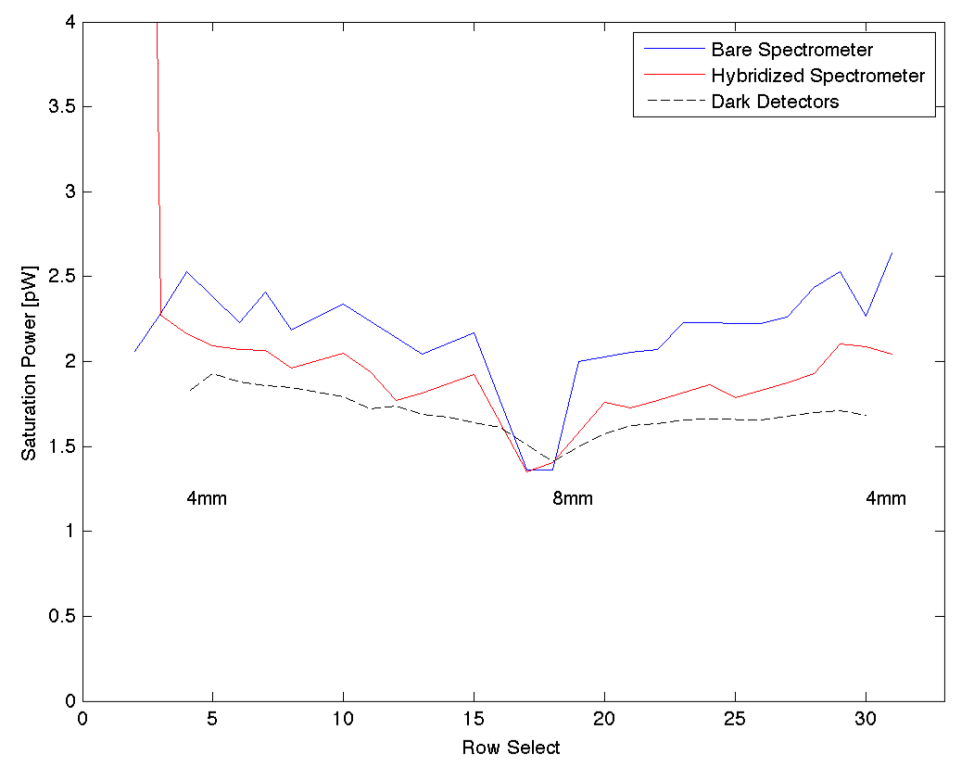

(a) Power received in each detector of three different devices defined in the text.

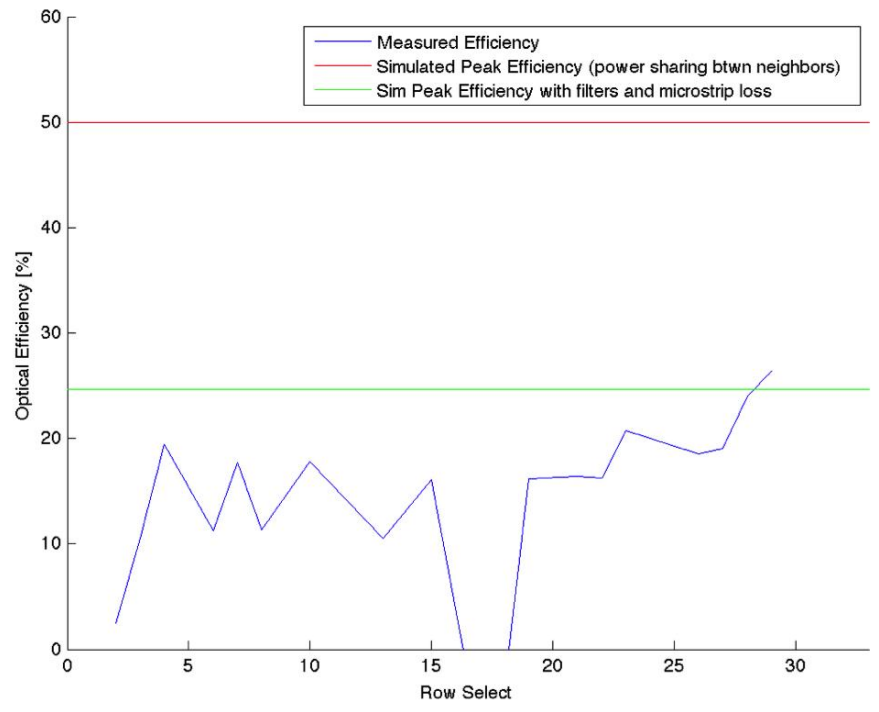

(b) Optical efficiency of directly fed spectrometer after correcting for dark power.

Figure 7. Received power and optical efficiencies for spectrometers

accounting for losses in the microstrip lines and filters, which will likely be improved in an optimized experiment. Test devices also suggest that the spectrometer itself performs with acceptable efficiency.

We are currently modifying our models to improve the agreement between theory and measurement. In particular, we are exploring HFSS simulations to model the thick film geometry rather than rely on empirically driven correction factors. These efforts are also aiming to better match the Qs on both sides of the resonators, so as to keep Q and efficiency high. We are also currently fabricating a full 80 channel version of our spectrometer that will omit the hybrid and simply combine currents with a 180 degree phase shift.

Our TDM readout system limits us to only a few modestly large spectrometers per tile. This limitation makes it difficult to fully explore the parameter space of design and material possibilities. For a TES-spectrometer to be viable in a field-able focal plane, we need to greatly increase our MUX factor in a way that limits the number of wire-bonds at the edge of our 


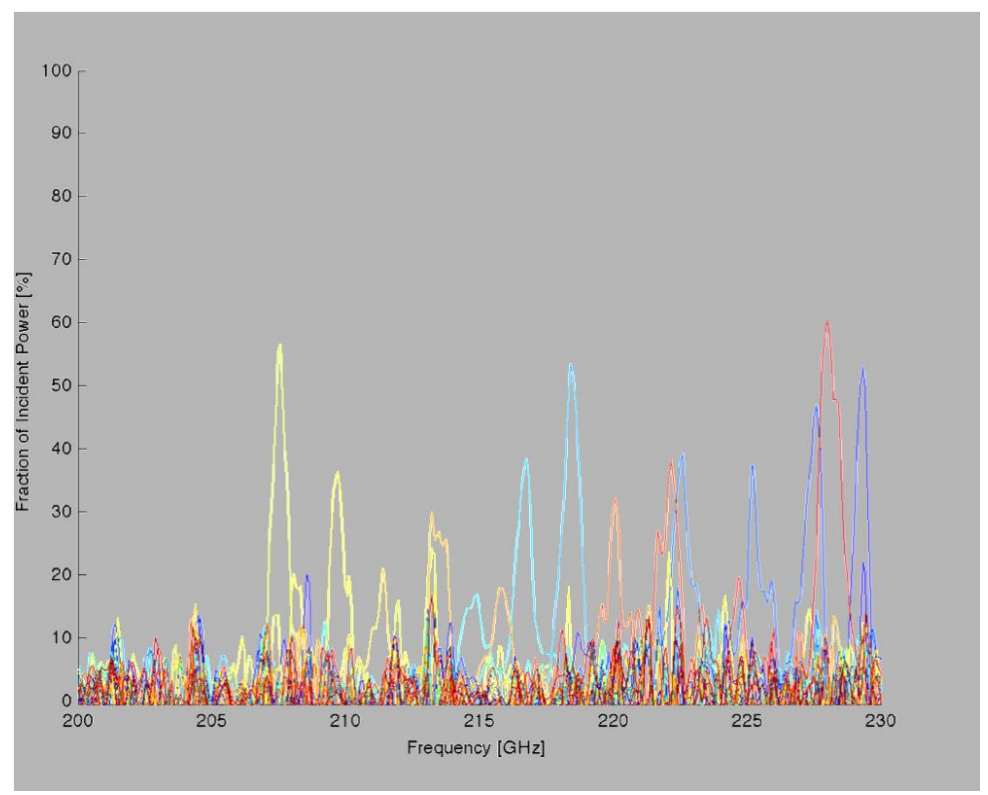

Figure 8. FTS spectra normalized to a reference bolometer behind a hybrid circuit.

tiles, and resonator-based readout is attractive. ${ }^{14}$ We plan to build future versions of these detectors that are compatible with this system in service of these goals.

\section{ACKNOWLEDGMENTS}

R. O'Brient would like to thank Oak Ridge Associated Universities for funding through the NASA Post-doctoral Program. All devices were fabricated in the Microdevices Laboratory (MDL) at NASA's Jet Propulsion Laboratory. We thank the Kavli Institute for Space Sciences (KISS) at Caltech for tomography study funding and funding for Abigail Crites in the KISS postdoctoral program.

\section{REFERENCES}

[1] G. D. Becker, W. L. W. Sargent, M. Rauch, and A. P. Calverley, "High-redshift Metals. II. Probing Reionization Galaxies with Low-ionization Absorption Lines at Redshift Six," ApJ 735, p. 93, July 2011.

[2] D. N. Spergel, R. Bean, O. Doré, M. R. Nolta, C. L. Bennett, J. Dunkley, G. Hinshaw, N. Jarosik, E. Komatsu, L. Page, H. V. Peiris, L. Verde, M. Halpern, R. S. Hill, A. Kogut, M. Limon, S. S. Meyer, N. Odegard, G. S. Tucker, J. L. Weiland, E. Wollack, and E. L. Wright, "Three-Year Wilkinson Microwave Anisotropy Probe (WMAP) Observations: Implications for Cosmology,” ApJS 170, pp. 377-408, June 2007.

[3] X. Fan, M. A. Strauss, R. H. Becker, R. L. White, J. E. Gunn, G. R. Knapp, G. T. Richards, D. P. Schneider, J. Brinkmann, and M. Fukugita, "Constraining the Evolution of the Ionizing Background and the Epoch of Reionization with z 6 Quasars. II. A Sample of 19 Quasars,” AJ 132, pp. 117-136, July 2006.

[4] A. Loeb and M. Zaldarriaga, "Measuring the Small-Scale Power Spectrum of Cosmic Density Fluctuations through $21 \mathrm{~cm}$ Tomography Prior to the Epoch of Structure Formation," Physical Review Letters 92, p. 211301, May 2004.

[5] C. L. Carilli, "Intensity Mapping of Molecular Gas During Cosmic Reionization," ApJL 730, p. L30, Apr. 2011.

[6] E. Visbal and A. Loeb, "Measuring the 3D Clustering of Undetected Galaxies Through Cross Correlation of their Cumulative Flux Fluctuations from Multiple Spectral Lines," in American Astronomical Society Meeting Abstracts \#218, p. 120.04, May 2011.

[7] S. C. Madden, A. Rémy-Ruyer, M. Galametz, D. Cormier, V. Lebouteiller, F. Galliano, S. Hony, G. J. Bendo, M. W. L. Smith, M. Pohlen, H. Roussel, M. Sauvage, R. Wu, E. Sturm, A. Poglitsch, A. Contursi, V. Doublier, M. Baes, M. J. Barlow, A. Boselli, M. Boquien, L. R. Carlson, L. Ciesla, A. Cooray, L. Cortese, I. de Looze, J. A. Irwin, K. Isaak, J. Kamenetzky, O. Ł. Karczewski, N. Lu, J. A. MacHattie, B. O”Halloran, T. J. Parkin, N. Rangwala, M. R. P. Schirm, B. Schulz, L. Spinoglio, M. Vaccari, C. D. Wilson, and H. Wozniak, "An Overview of the Dwarf Galaxy Survey," PASP 125, pp. 600-635, June 2013. 
[8] Y. Gong, A. Cooray, M. Silva, M. G. Santos, J. Bock, C. M. Bradford, and M. Zemcov, "Intensity Mapping of the [C II] Fine Structure Line during the Epoch of Reionization," ApJ 745, p. 49, Jan. 2012.

[9] Z. Staniszewski, J. J. Bock, C. M. Bradford, J. Brevik, A. Cooray, Y. Gong, S. Hailey-Dunsheath, R. O’Brient, M. Santos, E. Shirokoff, M. Silva, and M. Zemcov, "The Tomographic Ionized-Carbon Mapping Experiment (TIME) CII Imaging Spectrometer," Journal of Low Temperature Physics, May 2014.

[10] C. M. Bradford, P. A. R. Ade, J. E. Aguirre, J. J. Bock, M. Dragovan, L. Duband, L. Earle, J. Glenn, H. Matsuhara, B. J. Naylor, H. T. Nguyen, M. Yun, and J. Zmuidzinas, "Z-Spec: a broadband millimeter-wave grating spectrometer: design, construction, and first cryogenic measurements," in Z-Spec: a broadband millimeter-wave grating spectrometer: design, construction, and first cryogenic measurements, C. M. Bradford, P. A. R. Ade, J. E. Aguirre, J. J. Bock, M. Dragovan, L. Duband, L. Earle, J. Glenn, H. Matsuhara, B. J. Naylor, H. T. Nguyen, M. Yun, and J. Zmuidzinas, eds., Society of Photo-Optical Instrumentation Engineers (SPIE) Conference Series 5498, p. 257, Oct. 2004.

[11] A. e. a. Crites, "The time-pilot intensity mapping experiment," this proceedings , jul 2014.

[12] E. Shirokoff, P. S. Barry, C. M. Bradford, G. Chattopadhyay, P. Day, S. Doyle, S. Hailey-Dunsheath, M. I. Hollister, A. Kovács, H. G. Leduc, C. M. McKenney, P. Mauskopf, H. T. Nguyen, R. O’Brient, S. Padin, T. J. Reck, L. J. Swenson, C. E. Tucker, and J. Zmuidzinas, "Design and Performance of SuperSpec: An On-Chip, KID-Based, mmWavelength Spectrometer," Journal of Low Temperature Physics , Feb. 2014.

[13] S. Hailey-Dunsheath, P. S. Barry, C. M. Bradford, G. Chattopadhyay, P. Day, S. Doyle, M. Hollister, A. Kovacs, H. G. LeDuc, N. Llombart, P. Mauskopf, C. McKenney, R. Monroe, H. T. Nguyen, R. O’Brient, S. Padin, T. Reck, E. Shirokoff, L. Swenson, C. E. Tucker, and J. Zmuidzinas, "Optical Measurements of SuperSpec: A MillimeterWave On-Chip Spectrometer," Journal of Low Temperature Physics , Jan. 2014.

[14] J. A. B. Mates, G. C. Hilton, K. D. Irwin, L. R. Vale, and K. W. Lehnert, "Demonstration of a multiplexer of dissipationless superconducting quantum interference devices," Applied Physics Letters 92, p. 023514, Jan. 2008.

[15] S. e. a. O’Brient, R., "Antenna-coupled tes bolometers for bicep2 and keck array," in prep, tbd, jul 2014.

[16] BICEP2 Collaboration, P. A. R. Ade, R. W. Aikin, M. Amiri, D. Barkats, S. J. Benton, C. A. Bischoff, J. J. Bock, J. A. Brevik, I. Buder, E. Bullock, G. Davis, C. D. Dowell, L. Duband, J. P. Filippini, S. Fliescher, S. R. Golwala, M. Halpern, M. Hasselfield, S. R. Hildebrandt, G. C. Hilton, V. V. Hristov, K. D. Irwin, K. S. Karkare, J. P. Kaufman, B. G. Keating, S. A. Kernasovskiy, J. M. Kovac, C. L. Kuo, E. M. Leitch, N. Llombart, M. Lueker, C. B. Netterfield, H. T. Nguyen, R. O’Brient, R. W. Ogburn, IV, A. Orlando, C. Pryke, C. D. Reintsema, S. Richter, R. Schwarz, C. D. Sheehy, Z. K. Staniszewski, K. T. Story, R. V. Sudiwala, G. P. Teply, J. E. Tolan, A. D. Turner, A. G. Vieregg, P. Wilson, C. L. Wong, and K. W. Yoon, "BICEP2 II: Experiment and Three-Year Data Set," ArXiv e-prints, Mar. 2014.

[17] R. O’Brient, P. A. R. Ade, Z. Ahmed, R. W. Aikin, M. Amiri, S. Benton, C. Bischoff, J. J. Bock, J. A. Bonetti, J. A. Brevik, B. Burger, G. Davis, P. Day, C. D. Dowell, L. Duband, J. P. Filippini, S. Fliescher, S. R. Golwala, J. Grayson, M. Halpern, M. Hasselfield, G. Hilton, V. V. Hristov, H. Hui, K. Irwin, S. Kernasovskiy, J. M. Kovac, C. L. Kuo, E. Leitch, M. Lueker, K. Megerian, L. Moncelsi, C. B. Netterfield, H. T. Nguyen, R. W. Ogburn, C. L. Pryke, C. Reintsema, J. E. Ruhl, M. C. Runyan, R. Schwarz, C. D. Sheehy, Z. Staniszewski, R. Sudiwala, G. Teply, J. E. Tolan, A. D. Turner, R. S. Tucker, A. Vieregg, D. V. Wiebe, P. Wilson, C. L. Wong, W. L. K. Wu, and K. W. Yoon, "Antenna-coupled TES bolometers for the Keck array, Spider, and Polar-1," in Society of Photo-Optical Instrumentation Engineers (SPIE) Conference Series, Society of Photo-Optical Instrumentation Engineers (SPIE) Conference Series 8452, Sept. 2012.

[18] S. e. a. Ahmned, Z., "Bicep3: a 95ghz refracting telescope for degree-scale cmb polarization," this proceedings, jul 2014.

[19] A. S. Rahlin, P. A. R. Ade, M. Amiri, S. J. Benton, J. J. Bock, J. R. Bond, S. A. Bryan, H. C. Chiang, C. R. Contaldi, B. P. Crill, O. Doré, M. Farhang, J. P. Filippini, L. M. Fissel, A. A. Fraisse, A. E. Gambrel, N. N. Gandilo, S. Golwala, J. E. Gudmundsson, M. Halpern, M. F. Hasselfield, G. Hilton, W. A. Holmes, V. V. Hristov, K. D. Irwin, W. C. Jones, Z. D. Kermish, C. L. Kuo, C. J. MacTavish, P. V. Mason, K. Megerian, L. Moncelsi, T. A. Morford, J. M. Nagy, C. B. Netterfield, R. O'Brient, C. Reintsema, J. E. Ruhl, M. C. Runyan, J. A. Shariff, J. D. Soler, A. Trangsrud, C. Tucker, R. S. Tucker, A. D. Turner, A. C. Weber, D. V. Wiebe, and E. Y. Young, "Pre-flight integration and characterization of the SPIDER balloon-borne telescope," ArXiv e-prints , July 2014. 
[20] A. Goldin, J. J. Bock, C. L. Hunt, A. E. Lange, H. G. LeDuc, A. Vayonakis, and J. Zmuidzinas, "Design of broadband filters and antennas for SAMBA," in Millimeter and Submillimeter Detectors for Astronomy, T. G. Phillips and J. Zmuidzinas, eds., Society of Photo-Optical Instrumentation Engineers (SPIE) Conference Series 4855, pp. 163171, Feb. 2003.

[21] A. Orlando, R. W. Aikin, M. Amiri, J. J. Bock, J. A. Bonetti, J. A. Brevik, B. Burger, P. K. Day, J. P. Filippini, S. R. Golwala, M. Halpern, M. Hasselfield, G. Hilton, K. Irwin, W. C. Jones, M. Kenyon, C. L. Kuo, A. E. Lange, H. G. Leduc, B. Mates, T. Morford, H. T. Nguyen, R. W. Ogburn, C. Reintsema, M. Runyan, R. Sudiwala, A. Trangsrud, A. D. Turner, and P. Wilson, "Antenna-coupled TES Arrays For The BICEP2/Keck and SPIDER polarimeters," in American Institute of Physics Conference Series, B. Young, B. Cabrera, and A. Miller, eds., American Institute of Physics Conference Series 1185, pp. 471-474, Dec. 2009.

[22] P. Bodharamik, L. Besser, and R. Newcomb, "Two scattering matrix programs for active circuit analysis," Circuit Theory, IEEE Transactions on 18, pp. 610 - 619, nov 1971.

[23] S. e. a. Haily-Dunsheath, "Optical measurements of superspec: A sub/millimeter-wave on-chip spectrometer," this proceedings, jul 2014. 